\title{
The Evaluation Method for Operation Efficiency of Equipment System Based on Data Farming and Mining
}

\author{
Zheng Wang \\ College of Information System and Management, National University of Defense Technology, Changsha, Hunan \\ joshuakai900901@163.com
}

\begin{abstract}
Through process data and results data resulted from operation efficiency simulation of equipment system, based on information measure criteria, data farming and mining method is used. Final simulation results are provided against specific models and verification is carried out on the internal relationships of equipment system and the effectiveness of equipment tactics capability. In addition, its focus is made on utilizing high-performance calculations, running it in the whole parameter space of models, obtaining a number of effective sample space data, and further analyzing it to find implicit laws and results by Big Data Mining technology and Data Visualization technology, in order to facilitate the problem-oriented system efficiency simulation.
\end{abstract}

\section{Introduction}

Evaluation method research for operation efficiency of equipment system is an important part of equipment system demonstration. Due to the complexity of operation efficiency of equipment system, a new challenge is put forward to conventional operation simulation. Data Farming is used to study and analyze new technology of complex system. Data Farming technology provides new technical support for the operation efficiency evaluation of equipment system. On account of evaluation difficulties and features for operation efficiency of equipment system, it presents a new evaluation method for operation efficiency of equipment system based on the combination of Data Farming and Data Mining.

\section{Evaluation Difficulties and Features for Operation Efficiency of Equipment System}

Coordination operation efficiency evaluation under the joint operation conditions is required to analyze and assess the extent to achieve the target by all kinds of weapons or weapon system during joint operations and various operations [1]. Under the premise of joint operations becoming main operation type increasingly, the research on coordination operation efficiency evaluation has a great significance in the development of military construction and national defense industry, and can be used to overall optimize the use of existing equipment, assist in making military operations plans and decisions and provide a support to make new weapon system requirements and development programs [2] [3]. Besides, it can also support industrial department to do the trade-off research on weapon system improvement and modification [4], analyze the general design parameters of new models, review the system integrated scheme and so on.

The modeling simulation method and standardization research for coordination operation efficiency evaluation of equipment system centers on realistic needs of equipment information reform is also an innovation research platform of military theory to explore new concept and technology of information system operation. Meanwhile, the research is made on efficiency evaluation method of system-to-system confrontation operation requirements adaptive to information conditions, which is the representative core support technology of military complex system [5][6].

Currently, the modeling simulation method and standardization research for coordination operation efficiency evaluation of equipment system mainly consists of two parts: the modeling and simulation research is a more thorough study, but the modeling and simulation for coordination operation efficiency evaluation is a significant and challenging subject, which is a comparative weak part of research. Different from operation mode of a single conventional equipment or equipment system, the presence of operation efficiency of equipment system provides higher requirements for system coordination, mainly including: 1) Information is highly shared. Information share is to maximize the value of entity itself and use information to create new value. 2) Every mission is integrated. Although most time these missions belong to different entities in the system, in essence they are closely related. System is required to fully integrate all missions, in order to perform 
missions coordinately and smoothly. Just the coupling on the missions makes these entities form a coordination system. 3) All kinds of resources are deployed and optimized. As long as the system implements information share and mission integration, all kinds of resources in the system can break through various barriers and obstacles, in order to achieve a common target under unified management and coordination.

Multiple combat units perform combat missions jointly, which constitute a typical coordination system. Subject to the influence of various uncertainty factors of battlefield environment and the complexity of fleet size, the coordination operation process of multiple combat units is a complicated control problem, whose complexity mainly reflects the following aspects: the modeling complexity caused by the diversity of combat units and combat environment; the combination complexity caused by the amount growth of equipment and targets; the distribution complexity of combat units and the human complexity in multiple combat units. Relative to conventional operation simulation, operation efficiency evaluation of equipment system is more complicated and difficult. In addition, during the operation efficiency evaluation of equipment system, measurement is made qualitatively on the coordination between combat units, the emotive factors between command and control staff, etc.

However, in the conventional operation simulation, generation of cases is relatively difficult, the amount of available sample space is limited, it is helpful to verify the capacity of equipment system, but there is a big gap in the whole efficiency comprehensiveness of equipment system.

Therefore, there is a difficulty for the conventional operation simulation in the operation efficiency evaluation adaptive to equipment system. In recent years, constantly maturing data farming technology can generate a number of reliable sample data by farming data, while data mining technology is used to mine potential information in the database [7]. The combination of data farming technology and data mining technology can copy with the complexity in the operation efficiency evaluation of equipment system to some extent.

\section{Generation of System Simulation Sample Space Based on Data Farming Technology}

In 1996, Doctor Alfred G. Brandstein and Doctor Gary E. Horne proposed the data farming technology. It is a new problem-based technology that can provide effective solutions against problems of interest to user. The development of data farming technology is based on the following technical conditions:

a) The development and improvement based on agent model provides the possibility to master key elements during operations;

b) With the enhancement of computer capability, lots of analytical data can be produced;

c) The data organization, analysis and visualization capability is improved.

After Doctor Alfred G. Brandstein and Doctor Gary E. Horne developed the data farming technology, it was further complemented and improved by many scholars. It is now generally believed that the data farming technology mainly contains Fertilizing, Cultivating, Planting, Harvesting, Regenerating, etc. a) Fertilizing: Through mainly communicating with military researchers and associated experts, analysis is made on how to grasp some key elements during operations, but these elements used to be ignored, such as morale, command capability, intuition, etc.

b) Cultivating: Military experts put forward many views on key elements under specific battlefield environment and these views need to be analyzed and applied;

c) Planting: Expertise is used in the establishment of models, meanwhile the models are run, and the elements of interest are investigated and analyzed in a wide variable space;

d) Harvesting: Data generated by running models are collected, meanwhile some new technologies are developed and used to analyze these data;

e) Regenerating: On the basis of explaining and analyzing the farming results, next data farming is done under the guide of analysis results.

Above steps influence each other and have reference to each other, which form a whole data farming process, as shown in Figure 1.

Core concept of data farming is that through repeated calculation and constant feedback loop, a wide operation variable analysis space is obtained, in which user can excavate for answers to concerned problems. Moreover, in the variable analysis space, data farming especially attaches importance to the processing of outlier, and emphasizes the exploration of some key problems by the analysis of outlier. Above features are main differences between data farming technology and general operation simulation technology.

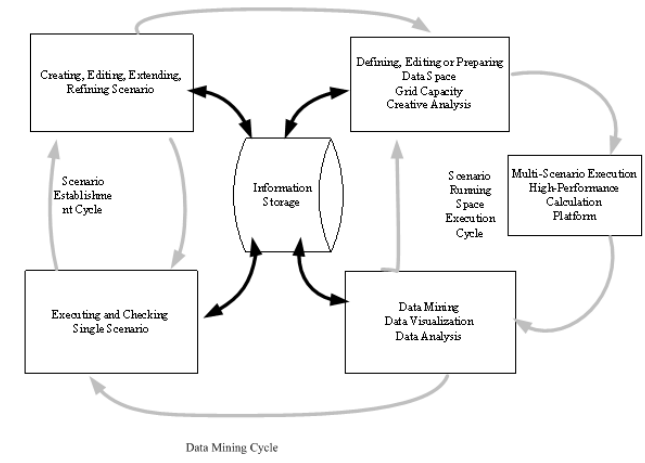

Fig. 1: Data Farming Process

Data farming is a reproductive method based on the problem analysis, i.e. the problem-based repeated train and data acquisition and analysis. This method can generate a lot of acceptable approximate values that are effective to answer studied problems. Data farming is not to use a specific model to give final answers, but to study the possibility space of answers to problems from a holistic perspective, and to transform conventional analysis methods and research ideas. In essence, it is to use high-performance calculations, run it in the whole parameter space of models, and gain hidden clues that cannot be obtained directly. The basic idea is to generate as much information as possible in the area of concern, use visualization, data mining and other methods to analyze data in specific sample space, obtain answers to problems of concern and its development trend, and guide the next farming. For problems of interest, users begin to generate data based on high-performance, including Fertilizing, Cultivating, Planting; Harvested data is analyzed by means of mining, visualizing and so on; finally, obtained results are stored to guide the next farming, i.e. implementing the 
regenerating process of farming. Through the cycle repetition, answers to problems of concern by user are gained. For example, for the simulation model of a fighter fleet based on Analytic Hierarchy Process (AHP), data farming is used to obtain data (normalization processed), as shown in table 1. Also, data farming process is a closed loop with analysis and verification, thus the reliability of obtained sample data based on data farming is guaranteed.

Table 1: Farming and Harvesting the Performance Index Of The Fighter Fleet

\begin{tabular}{|c|c|c|c|c|c|c|c|}
\hline Normalized Index & 1 & 2 & 3 & 4 & 5 & 6 & 7 \\
\hline Situation cognition quality & 0.843 & 0.848 & 0.862 & 0.873 & 0.892 & 0.910 & 0.917 \\
\hline Decision-making time & 0.672 & 0.663 & 0.691 & 0.682 & 0.695 & 0.624 & 0.683 \\
\hline Information acquisition capability & 0.878 & 0.862 & 0.891 & 0.885 & 0.902 & 0.856 & 0.883 \\
\hline Information distribution capability & 0.786 & 0.842 & 0.792 & 0.845 & 0.746 & 0.867 & 0.891 \\
\hline Information protection capability & 0.787 & 0.702 & 0.702 & 0.690 & 0.791 & 0.675 & 0.658 \\
\hline Coordination reliability & 0.823 & 0.832 & 0.834 & 0.823 & 0.845 & 0.817 & 0.825 \\
\hline Coordination credibility & 0.889 & 0.901 & 0.875 & 0.886 & 0.903 & 0.872 & 0.863 \\
\hline Target detection probability & 0.740 & 0.772 & 0.709 & 0.700 & 0.724 & 0.673 & 0.712 \\
\hline Target acquisition probability & 0.532 & 0.459 & 0.527 & 0.541 & 0.519 & 0.473 & 0.509 \\
\hline Probability of kill & 0.583 & 0.571 & 0.585 & 0.498 & 0.519 & 0.536 & 0.572 \\
\hline
\end{tabular}

\section{Application of Big Data Mining In Simulation-Based Efficiency Evaluation Of Equipment System}

Data farming technology resolves the sample space covering insufficiency problem in the operation efficiency evaluation simulation of equipment system. As performance or index of complex equipment system is numerous, by virtue of an evaluation model usually only using part of equipment data, or facing a type of evaluation targets, it is difficult to establish a unified evaluation model to describe the equipment comprehensively. When evaluating the equipment system, more and more elements need to be considered. For example, there are many indices of missile defense platform, performances vary in different territory, weather and application conditions, and many factors need to be considered to evaluate its operation performance. These element data describe the equipment system and its environment subtly, but the system evaluation model or algorithm is also required to make full use of these data, in order to generate more accurate results.

The grasp of comprehensive data is essential to provide accurate evaluation results of equipment system. With the development of information technology, there are more and more types of equipment represented by the information equipment, and system structure are more and more complicated, thus it is gradually difficult for conventional system evaluation method to process the equipment system with huge data. Big data mining technology can deal with huge data in the operation efficiency evaluation process of equipment system. Data mining is also called knowledge discovery in database, is a complex process that credible, novel, valuable modes or laws and other knowledge are extracted from huge data, and is one of means to analyze data farming results. It is simply explained to use software tools and database technologies to preprocess data and seek for useful hidden information and clues from lots of existing data. It is a integrated information processing technology that applies the machine learning into large database. The data mining process is shown as Figure 2. This process can use six basic steps to define, as follows:

a) Defining problems

b) Preparing data

c) Browsing data

d) Generating model

e) Browsing and verifying model

f) Deploying and updating model

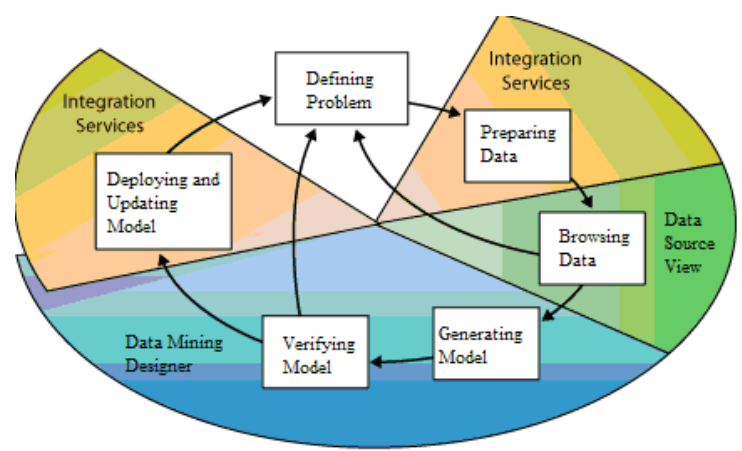

Figure. 2: General data mining process

For a specific complex military problem research, data farming method can be used to run lots of military models to obtain huge data. The database-based analysis on these huge data is the application of data mining technology. In accordance with interestingness of user, data is searched, merged and focused; Data miner is used to characterize, associate, classify and cluster data; the whole data mining process is a repeated iterative cycle that every technology is used to detect the small differences of data and different technologies and methods are used to constantly make the cycle analysis to get better or different results.

For example, in allusion to BMS simulation system in section 2, data is obtained by data farming. Data mining technology can be used to excavate BMS system 
characteristics hidden in data. According to different efficiency evaluation needs, different data mining algorithms are used respectively, such as characterization, association, classification, cluster, etc. For instance, we can use the association algorithm of data mining, such as Apriori algorithm, to find the association relationship between data 。

\section{Case Study}

Data farming and data mining are used to implement the operation efficiency evaluation of equipment system, as shown in Figure 3.

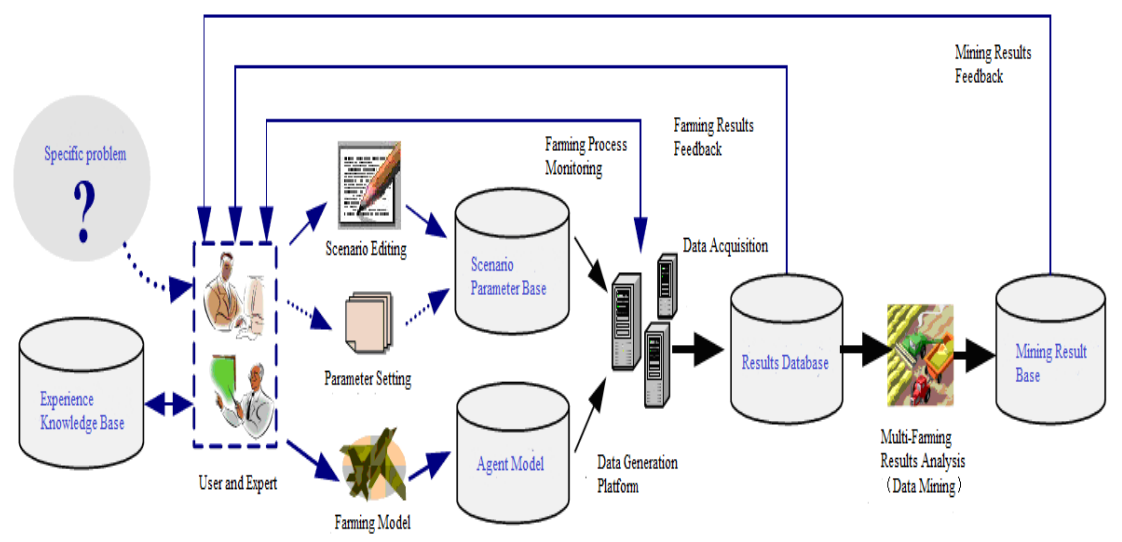

Figure. 3: Operation efficiency evaluation process of equipment system based on Data farming and data mining

The efficiency evaluation for coordination operation system of fighter fleet is still taken as an example. In section 2, data farming can be used on the coordination operation system of fighter fleet to obtain data, as shown in Table 1. Based on data farming, Apriori algorithm can be used to obtain the association relationship between every column of data in Table 1, as shown in Table 2. Table 2 shows that decision-making time is associated with information acquisition capability, information protection capability is associated with coordination reliability, etc.

These conclusions can help us to adjust the model of BMS simulation system to better make the efficiency evaluation of BMS system. For the same reason, other different data mining algorithms, such as neural networks, support vector machine etc., are used to excavate all kinds of information hidden in data mining results database, and provide the simulation for users and experts, to further adjust the simulation model.

\section{Conclusion}

This paper presents an evaluation method for operation efficiency of equipment system based on data farming and mining. Compared with conventional operation simulation method, its focus is on utilizing the data farming technology, running it in the whole parameter space of models, obtaining a number of effective sample space data, and further analyzing it to find implicit laws and results by Big Data Mining technology and Data Visualization technology, in order to facilitate the problem-oriented system efficiency simulation. This method has a certain reference value for the accurate evaluation on operation efficiency of equipment system by the use of small sample experiment data.

\section{References}

[1] Y. Liu, Research on the Passive Radar Guidance Technique and its Efficacy Evaluation Method under Confrontation Condition. Doctoral dissertation. National University of Defense Technology, 2010.
[2] J. Wang, Y. Liu, B. Deng, S. Geng, Analysis of Jamming Effectiveness against Early Warning Aircraft Tracking System. Journal of Naval University of Engineering, 2012.

[3] Chevalier, Tomas R., and Ove K. Steinvall. "Laser radar modeling for simulation and performance evaluation." SPIE Europe Security+ Defence. International Society for Optics and Photonics, 2009.

[4] Ewing, T., \& Tentner, A, Using a scalable modeling and simulation framework to evaluate the benefits of intelligent transportation systems (No. ANL/TD/CP-101364). Argonne National Laboratory, Argonne, IL (US), 2000.

[5] Patenaude, A., Study on the Effectiveness of Modeling and Simulation in the Weapon System Acquisition Process. OFFICE OF THE SECRETARY OF DEFENSE WASHINGTON DC, 1996.

[6] Ender, T., Leurck, R. F., Weaver, B., Miceli, P., Blair, W. D., West, P., \& Mavris, D. Systems-of-systems analysis of ballistic missile defense architecture effectiveness through surrogate modeling and simulation. IEEE Systems Journal, 4(2), 156-166, 2010.

[7] R. Ju, The research on the system evaluation method based on data farming and data mining. Doctoral dissertation. National University of Defense Technology, 2006. 